\author{
Alessandra Argenti Tremul \\ Centro di ricerche storiche di Rovigno (collaboratore esterno) \\ Piazza Matteotti 13, HR-52210 Rovigno - Rovinj \\ alessandra.atremul@gmail.com
}

\title{
Il Capodistriano del dopoguerra nelle fonti d'archivio conservate in Slovenia e Croazia
}

\author{
Pregledni rad | Review \\ UDK 94(497.1)"1945/1956":930.25(497.4) \\ Primljeno: 11. I. 2012.
}

\section{Sintesi}

In questo articolo viene delineata la situazione attuale degli archivi, che in Slovenia e Croazia raccolgono materiali relativi al periodo che va dal 1945 al 1956, che si riferiscono principalmente all'amministrazione jugoslava, quando l'area dell'Istria nord-occidentale era ancora contesa tra Italia e Jugoslavia, nonché alla successiva inclusione allo stato socialista jugoslavo.

\footnotetext{
Abstract

The author explains the main contents of the files, which are today available in the local and national archives of Slovenia and Croatia, regarding the Yugoslav administration of the zone B of the Free Territory of Trieste, from 1945 to 1956, and the first years after the inclusion of the northern Istria in the new socialist State.

Parole chiave: Istria, Litorale sloveno, Novecento, Territorio Libero di Trieste, amministrazione jugoslava, comunità nazionale italiana, fonti Key words: Istria, Slovene Coast, 20th century, Free Territory of Trieste, Yugoslav administration, Italian population, original documents
} 
Oggi è disponibile agli studiosi una copiosa documentazione riguardante il territorio classificato quale Litorale sloveno ovvero la parte nord-occidentale dell'Istria, nel periodo che va dal 1945 al 1956. Le istituzioni che conservano queste raccolte di carte, che ci sono pervenute fino ad oggi, relative al periodo in cui avvennero la costruzione del potere popolare e l'annessione del Capodistriano alla Jugoslavia, sono l’Archivio Regionale di Capodistria e l'Arhiv Republike Slovenije (Archivio della Repubblica di Slovenia) che si trova a Lubiana.

Di particolare interesse è il fondo intestato al "Comitato popolare circondariale dell'Istria”, cioè l'amministrazione civile dell'area contesa di fatto in mano jugoslava e che comprendeva i territori del Buiese e del Capodistriano dal 1947 al 1952, cioè dall'entrata in vigore del Trattato di pace con l'Italia, che oltre a cedere gran parte dei territori istriani alla Jugoslavia, istituiva il Territorio Libero di Trieste (di seguito TLT) a sua volta suddiviso in zona A e in zona B. Nel 1952 terminò la gestione unica dei distretti di Capodistria e Buie, siccome questi vennero fatti dipendere direttamente da Lubiana e Zagabria. Cessava così di esistere la "Circondariale" - così era chiamato dai funzionari dell'epoca l'organismo che raccoglieva i rappresentanti e i governanti di nazionalità slovena, croata e italiana della zona B del TLT. Questo fondo è catalogato e conservato presso l'Archivio regionale di Capodistria. È composto da molte sezioni: presidenza e segreteria, economato, cultura, sanità e tutela sociale, lavoro, giustizia, ufficio prezzi, infrastrutture e traffico, pianificazione territoriale, commercio, redazione del bollettino d'informazione, commissione per le trasgressioni, turismo, commissione di controllo, industria e artigianato, agricoltura, tribunale disciplinare, ufficio del personale, liste delle retribuzioni, organizzazione di partito. Da ciò si evince l'organizzazione primaria dell'amministrazione civile della zona B del TLT, guidata dal suo presidente Julij Beltram. Sono interessanti le buste relative all'organizzazione di partito (dal numero 172 al numero 176), in quanto si occupano della costruzione del potere popolare, cioè dell'assetto delle nuove strutture amministrative del territorio. Per lo studio della penisola istriana e delle sue trasformazioni occorse nel XX secolo, sembra utile anche la sezione che si occupava della cultura, ulteriormente suddivisa in slovena, italiana e croata (buste numero $38-63$ ). È necessario porre in rilievo come in questa prima fase del periodo post bellico, si ponesse molta attenzione a un'equa rappresentanza tra le varie nazionalità presenti sul territo- 
rio, come le tre lingue usate (italiano, sloveno e croato) fossero considerate paritetiche, e si cercasse di distribuire le risorse, di cui si disponeva, tra tutti e tre i gruppi linguistici, al fine del mantenimento e dello sviluppo delle istituzioni scolastiche, lo svolgimento di una certa attività culturale, che naturalmente all'epoca poteva essere solo di tipo progressista. Abbiamo trovato anche diverse buste inerenti gli indennizzi elargiti alle vittime del fascismo, che furono in qualche modo risarciti dei torti subiti in gran parte con i beni immobili sequestrati ad un gruppo non ancora ben definito di persone. Il Comitato popolare circondariale dell'Istria, aveva a tale scopo istituito una specie di cassa o fondo di assistenza e soccorso alle vittime del fascismo, gestito da Dante Crollini, futuro presidente dell'Unione degli Italiani dell'Istria e di Fiume, sezione del Capodistriano.

Sempre per quanto riguarda questo periodo di gestione comune del territorio in "temporanea" amministrazione jugoslava, possiamo trovare i documenti del Comitato popolare circondariale dell'Unione antifascista italo slava (di seguito UAIS), per il Litorale sloveno. Questo rientrava nella più ampia UAIS, l'organizzazione di massa, fondata a Trieste nell'agosto del 1945, che aveva lo scopo di riunire le diverse popolazioni che vivevano nella Regione Giulia e sulla base dell'unità e della fratellanza chiedere l'annessione del territorio conteso, dunque Trieste, il Goriziano, parte del Friuli e l'Istria alla Jugoslavia.

Vasta e corposa la collezione di documenti che si riferisce al Comitato popolare distrettuale di Capodistria $(1945$ - 1965). Segnaliamo i materiali che ci permettono di ricostruire la mappa del potere come ad esempio le liste dei segretari e dei presidenti delle comunità locali e dei comitati popolari cittadini, nonché delle persone elette nei vari comitati, dunque possiamo trovare i risultati elettorali del periodo tra la fine del secondo conflitto mondiale e l'istituzione del TLT (busta 149 A 29, 189 A 39). Per quanto riguarda il 1947 è disponibile pure l'elenco di tutti i funzionari pubblici impiegati presso il distretto di Capodistria (busta 180 A 37). In varie cartelle di questo fondo sono raccolti pure i verbali delle riunioni che si svolgevano nella sede del distretto.

Per quanto riguarda l'anno di svolta del 1948, quando avvenne la rottura tra la Jugoslavia ed il Cominform, notiamo uno stretto controllo sui funzionari pubblici che vengono schedati. Lanno dopo sono fatti fare pure degli esami e stilata una lista delle caratteristiche e delle note bio- 
grafiche per i singoli impiegati nell'amministrazione locale. Una struttura amministrativa, questa, che è riorganizzata nel 1949 dal nuovo segretario del Distretto di Capodistria, che manterrà tale carica fino alla soppressione di questa unità amministrativa, avvenuta nel $1965 .{ }^{1}$ La nuova sistemazione prevedeva sei gruppi principali di attività e cioè: le carte della presidenza, le finanze, l'economia (a sua volta suddivisa nelle sezioni approvvigionamento, industria, opere di ricostruzione, agricoltura, coordinamento e controllo dei prezzi), la giustizia comprensiva delle sentenze della commissione per le confische, l'assistenza sociale e la sanità, infine la scuola slovena, italiana e croata. Disponiamo di dettagliate liste del personale impiegato nella pubblica amministrazione anche per gli anni 1951 (busta 391 A 163) e 1953 (535 A 275).

Passiamo poi alle elezioni del 16 aprile 1950. Importanti perché furono le prime che si tennero in zona B. Erano state più volte rimandate a causa dell'incertezza dei risultati, o meglio la dirigenza comunista slovena dell'epoca temeva una grande disfatta. Infatti, nonostante un massiccio coinvolgimento dei mezzi di propaganda, quali la stampa e i programmi di Radio Capodistria, ci fu una bassissima affluenza alle urne. La popolazione votò appena nel pomeriggio dopo una serie di pressioni e l'allontanamento della stampa estera. Possiamo dunque oggi reperire i dati delle persone che furono elette in questa occasione nella busta 372 A 148.

Le circoscrizioni elettorali del distretto di Capodistria furono ridisegnate nel 1952 (b. 444 A 205). Abbiamo poi trovato l'elenco elettorale predisposto nel 1953, per scegliere i rappresentanti nella camera del lavoro.

La suddivisione amministrativa del Litorale sloveno venne ovviamente modificata nel 1954 e nel 1955 (bb. 540 A 275, 1022 A 718), contemporaneamente alla soluzione politica del contenzioso confinario tra Italia e Jugoslavia, con la spartizione del TLT, sancita dalla firma del Memorandum di Londra. Ci furono poi dei programmi d'investimento specifici che accompagnarono l'annessione di questa zona alla Jugoslavia (bb. 665-672). Furono inoltre preparate delle nuove versioni dello statuto comunale di Capodistria, che all'epoca era la cittadina più importante di quest'area dove era concentrata l'amministrazione di tutta l'area dell'Istria nord-occidentale, alcune piccole industrie, fungeva da centro culturale e proprio qui stava nascendo

1 Si tratta di Črtomir Kolenc. Per una sua biografia cfr. Primorski slovenski biografski leksikon, 8, Gorica 1982, 103-104. 
quello scalo marittimo, molto desiderato dalle autorità e dal partito comunista sloveni, che in qualche modo avrebbe dovuto compensare la perdita della città di Trieste e del suo porto.

L'articolo 8 del Memorandum d'intesa tra i governi d'Italia, del Regno Unito, degli Stati Uniti e di Jugoslavia, relativo al Territorio Libero di Trieste, siglato a Londra il 5 ottobre 1954, dava la possibilità alle persone presenti in tale area di lasciare la propria residenza entro un anno dalla parafatura di tale accordo e di trasferire i propri beni mobili e immobili. ${ }^{2}$ A tale proposito va rilevato che in questo fondo sono conservati tutti i permessi rilasciati, nel 1955 e nel 1956, dal Segretariato per gli affari interni del Comitato popolare distrettuale di Capodistria. Questi sono stati ampiamente analizzati da Jure Gombač. ${ }^{3}$

Delle nuove elezioni si tennero nel 1957 (b. 972 A 689). Di fatto il Capodistriano ora è completamente inserito nella realtà jugoslava, il suo quadro demografico completamente cambiato, tanto che le autorità governative registrano l'effettiva nascita della minoranza italiana.

Il fondo relativo al distretto di Capodistria si è rivelato prezioso per quanto riguarda la conoscenza della comunità nazionale italiana, siccome conserva una analisi dettagliata di questa parte della popolazione istriana che nel 1957 viene effettivamente registrata in qualità di minoranza nell'Istria nord-occidentale. Tale documentazione (1994 A 1441), appartenuta al segretario del distretto, raccoglie anche i materiali della commissione mista italo-jugoslava che in base alle disposizioni dello Statuto Speciale annesso al Memorandum di Londra, doveva sorvegliare l'attuazione dei diritti concessi al gruppo etnico jugoslavo vivente in Italia e a quello italiano rimasto in Jugoslavia ${ }^{4}$ (1995 A 1442, 1443).

Appare invece molto promettente la busta contenente gli inventari delle aziende della ex zona B del TLT. In realtà è stata una delusione: una volta aperta non è stato trovato praticamente nulla (1796 A 1351). Sembra sia andata persa anche la documentazione della Banca d'Istria seppure sia presente nel

\footnotetext{
Manlio Udina, Gli Accordi di Osimo, Trieste 1979.

Jure Gombač, Esuli oppure optanti?, Trieste 2007.

Come leggiamo nel punto 8 dello Statuto speciale, Allegato II al "Memorandum d'intesa tra i governi d'Italia, del Regno Unito, degli Stati Uniti e di Jugoslavia, relativo al Territorio libero di Trieste" (Londra, 5 ottobre 1954): "A special Mixed Yugoslav-Italian Commitee shall be established for the purpose of assistance and consultation concerning problems relating to the protection of the Yugoslav ethnic group in the area under Italian administration and of the Italian ethnic group in the area under Yugoslav administration." Udina, Gli accordi di Osimo, 142.
} 
catalogo dell'Archivio regionale di Capodistria. ${ }^{5}$ Un vero peccato siccome qui dovrebbero essere raccolti molti dati di fondamentale importanza (ad esempio sui conti del potere popolare, sulle imprese, sui depositi o quant'altro di utile per capire la situazione economico-finanziaria) visto che perlomeno fino al 1952 era l'unica esistente nell'area da noi presa in considerazione.

A Zagabria abbiamo trovato due piccoli fondi depositati presso il Hrvatski državni arhiv (Archivio di Stato Croato). Sono in tutto quattro preziose buste, che raccolgono una serie di documenti della Amministrazione militare dell'Armata jugoslava nel Territorio Libero di Trieste (VUJA), che aveva sede a Capodistria e gestiva tutta la zona B del TLT (dunque il Capodistriano e il Buiese). In questi incartamenti possiamo trovare i bilanci del Comitato popolare circondariale per l'Istria, di cui abbiamo detto poco sopra, dai quali si evince la situazione economica, ma anche le reali necessità della zona B. A destare la nostra curiosità è stato ad esempio un elenco dettagliato della quantità di scarpe necessarie in tale area ordinate dalla Circondariale e pagate dal governo militare jugoslavo. Restano però un mistero i criteri di distribuzione ovvero la lista degli aventi diritto. In base a tali liste osserviamo che ce ne sono anche della misura adatta ai bambini.

Di notevole interesse sono i verbali delle riunioni congiunte tra i rappresentanti della VUJA e quelli del Governo militare alleato, che invece gestiva la zona A compresa Trieste, inerenti i problemi più scottanti dell'intero TLT. Questo argomento per la sua delicatezza meriterebbe certamente una trattazione specifica.

Altre due buste si riferiscono invece al Tribunale dell'Amministrazione militare jugoslava per la Regione Giulia, l’Istria, Fiume e il Litorale sloveno, che aveva sede ad Abbazia ed operò dal 1945 al 1947.

A Lubiana, presso l'Archivio della Repubblica di Slovenia, abbiamo scandagliato innanzitutto gli archivi, che originariamente facevano parte dell’Archivio Storico della Lega dei Comunisti della Slovenia. Qui ci sono molti fondi interessanti, per il momento abbiamo guardato quelli che fanno riferimento al massimo livello decisionale, cioè al Comitato centrale del Partito comunista sloveno (di seguito CC PCS). Di fondamentale impor-

5 Qui rileviamo un fatto curioso, qualche tempo dopo aver concluso il presente saggio, le carte di cui in oggetto sembra siano riapparse nella loro collocazione originaria, ma purtroppo per motivi di tempo non abbiamo potuto controllare il tutto personalmente. 
tanza è l'immenso materiale raccolto nei fondi del Ministero degli Interni. Questi essendo molto vasti e articolati, siccome riguardano le istituzioni che di fatto gestivano tutta l'area in mano alla Repubblica Socialista di Slovenia, coprono moltissimi argomenti e tutte le località del Paese dalla fine della Seconda guerra mondiale alla morte della Jugoslavia. Proprio per questa loro ampiezza la ricerca è tutt’altro che facile.

Per quel che riguarda la documentazione del CC PCS sono reperibili alcune relazioni sulla situazione politica del Litorale sloveno che ci permettono di analizzare dei particolari momenti, come ad esempio gli anni 1948 e 1949, problematici a causa del noto dissidio tra la Jugoslavia ed il Cominform. Questa rottura ebbe conseguenze notevoli anche sul territorio istriano siccome scatenò non solo tutta una serie di scelte e divisioni, ma dette il via a molte di ripercussioni e il nascente Potere Popolare fece sentire la sua forza, anche togliendo la libertà a chi la pensava in maniera diversa. Ciò scatenò una vera e propria caccia alle streghe, che in Istria colpì soprattutto gli appartenenti alla Comunità Nazionale Italiana apertamente schierati per l'internazionalismo comunista e dunque in contrasto con la linea titoista, molti finirono a Goli otok. Tali spaccature sono ben visibili in zona B dove il Partito comunista del TLT, che fino a quel momento aveva compreso italiani, sloveni e croati nella comune lotta contro l'imperialismo, si scinde in due fazioni. La parte guidata da Vittorio Vidali (legata all'Internazionale comunista) era sostenuta in gran parte dagli italiani, mentre quella di Branko Babič era collegata direttamente al Partito comunista sloveno e a quello jugoslavo. Da questo momento in poi ci fu una vera e propria lotta senza tregua tra le due fazioni - soprattutto in zona A, che si dividono tutto, non solo i tesserati ma anche le proprietà e le risorse che fino a quel momento avevano avuto in comune. Nella zona B per qualche anno ancora operava una sezione del Partito Comunista Italiano. ${ }^{6}$

È possibile ottenere un quadro generale della situazione in Slovenia e in parte anche per l'Istria nord-occidentale, attraverso le preziose trascrizioni dei verbali del Politbiro del CC PCS, ora raccolte nel volume edito dall'Archivio di Stato Sloveno a cura di Darinka Drnovšek. ${ }^{7}$

Naturalmente, per quanto riguarda il territorio istriano è utile indagare anche i fondi del Comitato centrale e di quello circondariale del PC della Regione Giulia e del Territorio Libero di Trieste.

\footnotetext{
6 Cfr. in merito Paolo Sema, El Maestro de Piran, Tricesimo 1999.

7 Darinka Drnovšek (a cura di), Zapisniki politbiroja CK KPS/ZKS 1945-1954, Viri 15, Ljubljana 2000.
} 
Per quanto riguarda le organizzazioni di massa tipiche del periodo socialista, sempre a Lubiana è disponibile, anche se non completamente catalogato, il fondo relativo alla Alleanza socialista del popolo lavoratore (di seguito ASPL) meglio nota come la SZDL. Ci siamo limitati a guardare le buste relative alla presidenza della Conferenza repubblicana, dunque al massimo organo decisionale di questa mastodontica associazione che praticamente inglobava tutta la società dell'epoca del real socialismo jugoslavo, ma che era sorta appena nel 1953 . Nel periodo precedente alla creazione di questo massiccio organismo di massa, i vertici dell'ASPL corrispondevano al Comitato esecutivo del Fronte di liberazione, dunque alla Osvobodilna fronta (di seguito OF). Questo ristretto gruppo decisionale, con la fine della Seconda guerra mondiale, rimase in funzione all'interno del Fronte popolare della Jugoslavia. Oltre ad occuparsi delle maggiori necessità del paese quali ad esempio l'introduzione del sistema economico pianificato, il cooperativismo, la lotta al clero e alle forze reazionarie straniere, si occupava anche dei territori considerati sloveni ma non ancora inclusi nella madrepatria, cioè la Carinzia, Trieste e il Litorale sloveno. In questi otto anni ne fecero parte le massime cariche dello Stato, dal presidente dell'Assemblea Popolare della Repubblica Popolare Slovena, dal capo del governo a vari alti funzionari del PCS, che si occupavano delle singole tematiche che venivano messe all'ordine del giorno. Per quanto riguarda l'area dell'Istria nord-occidentale, all'epoca territorio conteso tra l'Italia e la Jugoslavia, ne discutevano, oltre a chi effettivamente in quel momento lo stava amministrando per conto del governo sloveno, anche quelle persone che erano state direttamente coinvolte nelle varie fasi operative della battaglia per Trieste (b. 24, 02-8, 01-30).

Nelle riunioni dell'ASPL, infatti, si discute ampiamente della questione di Trieste. Queste furono particolarmente numerose nel 1953, in concomitanza con la cosiddetta "crisi di Trieste", e nel 1954, quando tale problema si chiuse con la firma del Memorandum di Londra. In base alla documentazione consultata risulta chiaramente che l'inclusione del distretto di Capodistria entro le strutture dello stato sloveno, dunque nella Jugoslavia, avvenne appena nel 1954 (b. 02.1/55).

Ci sono apparsi molto utili anche i fondi appartenuti ai due più importanti politici sloveni del dopoguerra, come pure della guerra stessa, Edvard Kardelj e Boris Kraigher, sempre conservati presso l'Archivio della Repubblica di Slovenia. 
La raccolta di carte appartenute a Kardelj, potente Ministro degli esteri jugoslavo e vicepresidente della Federativa, è stata una vera delusione, nel senso che ci aspettavamo di trovare dei materiali relativi alla questione delle minoranze, più precisamente di avere qualche notizia sul destino degli Italiani in Istria, nelle varie fasi delle trattative diplomatiche per la delimitazione del confine. Da quanto abbiamo potuto constatare non c'è nulla di rilevante rispetto alle vicende dell'Istria. Si tratta per lo più di fotocopie, di una scelta di documenti da lui redatti o da lui utilizzati. Per ora è ancora sconosciuta l'ubicazione esatta del suo vero archivio personale.

Al contrario, il fondo intitolato a Boris Kraigher, che fu non solo coinvolto in prima linea nella cosiddetta "corsa per Trieste", membro dell'esecutivo dell'OF, vicepresidente del Comitato regionale di liberazione nazionale per il Litorale sloveno, ministro degli interni della Repubblica Popolare Slovena, nonché presidente del Consiglio esecutivo dell'Assemblea popolare della Slovenia dal 1953 al 1962, è ricco. La documentazione raccolta è sistemata e redatta in maniera abbastanza dettagliata. Si possono trovare anche delle buone sintesi di quelli che erano i problemi più urgenti da affrontare, all'epoca, come lo potevano essere i rapporti tra Stato e Chiesa, i territori interessati da trattative diplomatiche in corso o la scissione del partito comunista del TLT in seguito alle controversie avute dal PCJ con il Cominform. A ciò si aggiungono una serie di cartelle dal carattere piuttosto personale.

Infine abbiamo tentato di trovare i documenti, oggi esistenti e non catalogati, che appartennero ad alcune persone che nel dopoguerra ebbero, a livello locale, un certo ruolo istituzionale. Questo è un lavoro molto difficile, in quanto dipende solo dalla buona volontà dei curatori o dei titolari o dei familiari che hanno ora in mano raccolte di carte all'apparenza secondarie, ma che qualche volta possono contenere proprio quei tasselli mancanti, ma necessari, per poter raccontare una determinata vicenda, oppure per capire l'evoluzione degli avvenimenti di un dato periodo. Qui non posso non ringraziare le signore Ondina Gregorich Diabaté e Clio Diabaté, che molto gentilmente mi hanno messo a disposizione una serie di documenti relativi al Comune e al Distretto di Capodistria, i quali si sono rivelati preziosi per la comprensione e lo sviluppo della attuazione del bilinguismo ovvero 
del sistematico ridimensionamento dell'utilizzo della lingua italiana in quest'area dalla fine della Seconda mondiale in poi.

Merita senz'altro di essere consultato il Centro di ricerche storiche di Rovigno (di seguito CRS), dove sono conservati molti documenti relativi alla presenza della comunità nazionale italiana autoctona in Istria, Fiume e Dalmazia, nonché una ricchissima collezione cartografica, una raccolta di giornali d'epoca, una splendida biblioteca specializzata su tematiche relative alla penisola istriana. Qui sono reperibili i documenti dell'Unione degli Italiani, cioè l'organizzazione di massa che raccoglieva (dal 1944 al 1991) gli italiani rimasti a casa proprio nonostante il passaggio ad una realtà statuale diversa dalla nazione madre, e che - secondo quando disposto dalle autorità del regime jugoslavo - ebbero il compito di costruire il nuovo stato socialista e la fratellanza con le popolazioni slovena e croata.

Per comprendere appieno il Novecento sono molto utili, anche se non usate tantissimo, le fotografie che ritraggono luoghi, avvenimenti, persone che si riferiscono ad un preciso periodo. Le fotografie ci danno un livello in più di informazioni o perlomeno ci aiutano a inquadrare meglio la situazione.

Le istituzioni che conservano dei bei archivi fotografici, per quel che riguarda il periodo dopo la Seconda guerra mondiale, sono il CRS di Rovigno e il Museo regionale di Capodistria. Il CRS ha delle collezioni davvero uniche che ci raccontano tutto il periodo dal 1945 ad oggi, con fotografie che riportano sia i principali avvenimenti e personaggi, sia la realtà locale.

Presso il Museo regionale di Capodistria invece, nella sua sezione dedicata all'epoca contemporanea, sono raccolte molte fotografie che si riferiscono al periodo del Territorio Libero di Trieste, in particolare alla società e agli avvenimenti della zona B. In questo modo sono documentati i raduni di massa, i volti dei principali esponenti politici, le varie ricorrenze e celebrazioni.

Presso l'Archivio della Repubblica di Slovenia abbiamo trovato dei filmati d'epoca, praticamente sconosciuti, che sono stati realizzati per documentare l'acquisizione di nuovi territori da parte della Jugoslavia. Con questa specie di cine-giornali si voleva far conoscere all'opinione pubblica 
jugoslava l’Istria e il Litorale sloveno, con chiari intenti di propaganda. Oppure semplicemente il potere costituito aveva intenzione di registrare e studiare questa nuova realtà.

Il materiale girato, non catalogato o analizzato fino ad ora, ci è parso molto interessante.

Nel filmato Filmski obzornik: Boj in žrtve ljudstva Julijske krajine za priključitev k Jugoslaviji [La lotta della popolazione della Venezia Giulia per l'annessione alla Jugoslavia] (1946) sono registrate le manifestazioni a favore della Jugoslavia che si tennero a Trieste nel 1946, presumibilmente quando era in corso la Conferenza di pace di Parigi. In questa massa di persone, oltre agli agenti della Polizia del Governo militare alleato (GMA), possiamo notare delle scritte in lingua slovena, poi vengono montate delle immagini relative alla zona jugoslava dove si vede la corsa con la staffetta, il ministro degli esteri Edvard Kardelj e la partenza, con il treno, della delegazione jugoslava, sulla cui locomotiva è affissa l'alabarda simbolo della città di Trieste, alla volta di Parigi, dove erano in corso le trattative diplomatiche seguite alla fine della Seconda guerra mondiale.

In Priključitev Istre in Slovenskega primorja k domovini [L'annessione dell'Istria e del Litorale sloveno alla madrepatria - b/n] si vedono i militari jugoslavi, dei comandanti, molta gente e cartelli inneggianti alla Jugoslavia, bandiere jugoslave, dei politici che tengono dei discorsi di circostanza, diverse scritte favorevoli all'annessione della Primorska alla Jugoslavia.

In Predvolilno zborovanje v Kopru [Il comizio elettorale a Capodistria - b/n] (1950) gli striscioni sono scritti in lingua italiana e pubblicizzano il Fronte popolare, cioè il partito ufficiale della zona B del TLT, sostenuto dall'amministrazione jugoslava. La piazza principale di Capodistria con il Palazzo Pretorio, la cui scalinata fa da palcoscenico, la Loggia e il Duomo, è gremita di gente. Sono presenti alcune immagini della simbologia comunista quali stelle rosse e quadri di Tito. C'è pure una scritta in sloveno a favore del cooperativismo e della riforma agraria. I discorsi sono tenuti da Branko Babič, che era il principale esponente del PC regionale, nonché da Ivan Regent, noto comunista sloveno. Un segno messo dalla nuova amministrazione per marcare il territorio su questa bellissima piazza rinascimentale sono dei grandi archi di foglie e piante, completamente estranei al mondo delle cittadine istriane di impronta veneta. 
Il corteo si sposta poi a Šmarje, paesino ubicato sulle colline alle spalle di Capodistria, dove si possono vedere tante donne slovene con le bandiere, un'urna elettorale, il corteo passa attraverso un arco di trionfo di piante, in mezzo alle case distrutte, si va a votare in massa nella casa del cooperatore.

In Na Okroglici [A Okroglica - b/n] (1953) i cineoperatori di stato rappresentano quella famosa manifestazione massmediologica in cui Tito prese una forte, ma anche preoccupante, posizione nei confronti dell'Italia. Siamo in piena "crisi di Trieste" e all'opinione pubblica si vuole mostrare un gruppo dirigente compatto e unito, che sta facendo l'unica scelta giusta. Sul palco vediamo infatti attorno al presidente della Jugoslavia, seppure per brevissimi istanti, il ministro degli esteri nonché vice-presidente della Jugoslavia Edvard Kardelj, il presidente della Assemblea popolare, nonché del governo della Slovenia, oltre a essere segretario del Comitato centrale del Partito Miha Marinko, il ministro degli interni Boris Kraigher, il massimo esponente del PCS a livello locale Branko Babič. La preziosità di questo filmato sta proprio in questo quadretto che ritrae quel gruppo di persone che ufficialmente si occupavano della cosiddetta "questione di Trieste", cioè del problema dell'acquisizione e dell'annessione del Litorale sloveno, dunque del territorio del Capodistriano, il cui destino all'epoca non era ancora del tutto chiaro.

Quanto questi momenti siano stati importanti per la classe dirigente nazionale lo deduciamo dal fatto, che questo documentario ha pure una seconda versione. Na Okroglici (colore, 1953) è pure il primo filmato a colori prodotto in Slovenia, dalla Triglav film di Lubiana, seppure la pellicola sia stata sviluppata in Germania per via della tecnologia altamente innovativa dell'Agfacolor. Da ciò possiamo dedurre la centralità del problema di Trieste per la politica e la società slovena di allora. Ci sono da mettere in rilievo alcune particolarità del film: innanzitutto manca il sonoro originale, ma c'è solo una voce maschile fuori campo che legge un testo cercando di raccontare nel modo più appropriato l'avvenimento, in cui è però chiaro l'atteggiamento anti-italiano di tale manifestazione. Viene dunque detto in modo esplicito che gli italiani non hanno alcun diritto su questi territori. Dall'altra parte nella sfilata dei gruppetti a cui è consentito salutare il maresciallo Tito, ci sono anche un paio di rappresentanti autorizzati della comunità italiana che hanno con sé la bandiera con la stella rossa, indossano il fazzoletto rosso al collo e la bustina sulla testa. 
In questo archivio abbiamo visto anche una serie di filmati che non sono stati classificati siccome non hanno un titolo e il contenuto sembra sconosciuto. Si tratta di materiale grezzo che risale alla fine degli anni Cinquanta e agli inizi degli anni Sessanta.

La nostra attenzione è stata subito catturata da alcune immagini marine. Visionando questi filmati abbiamo scoperto dei paesaggi, dimenticati dalla nostra memoria, ma che ci danno l'immagine di quelle che erano le cittadine istriane costiere prima dell'industrializzazione e della cementificazione, che ne hanno inesorabilmente cambiato il volto. Pirano, Isola e Capodistria dopo l'esodo appaiono semi-deserte, seppure conservino ancora la loro fisionomia tradizionale, i nomi delle imbarcazioni non sono più nomi di donna, le scritte solo in sloveno.

*

L'accesso a nuovi fondi d'archivio permette ai ricercatori di avviare indagini che certamente contribuiscono a delineare un quadro sempre più completo dell'Istria (e delle sue trasformazioni) del dopoguerra. A tale riguardo vanno prese in considerazione anche tipologie di fonti non ancora molto usate dalla storiografia attuale, come ad esempio i filmati d'epoca e le fotografie, ma che possono completare - a volte anche in maniera determinante - il racconto storico.

\section{Fonti}

Archivio regionale di Capodistria, F. Comitato popolare circondariale dell'Istria.

Archivio regionale di Capodistria, F. Comitato popolare circondariale dell'UAIS.

Archivio regionale di Capodistria, F. Comitato popolare distrettuale di Capodistria.

Arhiv Republike Slovenije (Archivio della Repubblica di Slovenia), F. SZDL (Alleanza socialista del popolo lavoratore), 537.

Arhiv Republike Slovenije, F. CC PCS - 1589(III), 1569.

Arhiv Republike Slovenije, F. Film d'epoca.

Arhiv Republike Slovenije, F. Edvard Kardelj - 1277.

Arhiv Republike Slovenije, F. Boris Kraigher - 1529. 
Arhiv Republike Slovenije, F. Ministero degli Interni della Repubblica Socialista di Slovenia - 1931.

Centro di ricerche storiche di Rovigno, F. Unione degli Italiani / zona B. Museo regionale di Capodistria, F. TLT.

\begin{abstract}
Riassunto
L'autrice, dopo aver visionato gli archivi regionali e nazionali, che conservano i fondi relativi all'amministrazione della zona B del Territorio Libero di Trieste, presenta una scelta delle raccolte analizzate. Queste possono venir oggi utilizzate per delineare in maniera sempre più chiara le vicende occorse nella penisola istriana dal 1945 al 1956, ed alcuni suoi momenti fondamentali come la rottura con il Cominform, l'amministrazione locale, il trasferimento di massa della popolazione autoctona. In questo periodo, dopo una lunga e plurisecolare permanenza sul proprio territorio d'insediamento storico, la popolazione italiana diventa minoranza. Un contributo importante allo sviluppo della storiografia regionale può essere dato pure da fonti di nuova tipologia, come ad esempio i filmati e le fotografie d'epoca, fino ad ora usate in parte minima.
\end{abstract}

\title{
Povojna Koprščina v arhivskih virih hranjenih v Sloveniji in Hrvaški Povzetek
}

Avtorica predstavlja izbor analiziranih zbirk gradiva iz pokrajinskih in državnih arhivov, ki hranijo fonde, ki se nanašajo na administracijo cone B Svobodnega tržaškega ozemlja. To gradivo lahko danes služi za še temeljitejšo obrazložitev dogodkov, ki so na istrskem polotoku v letih 1945 - 1956 privedli do spora z Informbirojem, do lokalne uprave in množične selitve avtohtonega prebivalstva. Po dolgi in večstoletni nastanjenosti na območju zgodovinske prisotnosti postaja namreč v tem času italijansko prebivalstvo manjšinsko. Pomemben prispevek k razvoju regionalnega zgodovinopisja nudijo tudi viri novejšega tipa, do sedaj uporabljeni le delno, kot so filmski in fotografski posnetki iz takratnega obdobja.

\section{Poslijeratna Koparština u slovenskim i hrvatskim arhivskim fondovima \\ Sažetak}

Nakon uvida u regionalne i državne arhive u kojima se čuvaju fondovi koji se odnose na upravu Zone B Slobodnog teritorija Trsta, autorica predstavlja izbor analiziranih zbirki. One mogu danas biti korištene za jasnije ocrtavanje događanjā na Istarskom poluotoku između 1945. i 1956. i nekih njihovih temeljnih točaka poput raskida s Informbiroom, mjesne uprave i masovnog iseljavanja autohtonog stanovništva. U to je doba, nakon višestoljetnog obitavanja na svom matičnom području, talijansko stanovništvo postalo manjinom. Važan doprinos razvoju regionalne historiografije mogli bi dati izvori novoga tipa, poput filmova i fotografija toga vremena, do danas vrlo malo korišteni. 


\section{The Koper region after the war in Croatian and Slovenian fonds}

\section{Summary}

The national and regional archives of Slovenia and Croatia have a lot of documents regarding the Yugoslav administration of the Free Territory of Trieste. The author illustrates a choice of the main files that nowadays are very useful for writing the history of the Istrian peninsula from the 1945 to the 1956. Historians can now study new arguments regarding the Istrian peninsula after the Second World War, such as the development of the local administration, the consequences after the problems between Tito's Yugoslavia and Stalin's USSR, and so on. In that time the Italian population, who has lived in the northern Istria for centuries became a minority. It could be very important to use all the information on this subject that we can find in old photographs and films when researching history. A new approach for writing history, which could be very interesting. 
